\title{
Machine Learning Application in Process on Extra Virgin Olive Oil Elaboration Disk Stack Vertical Centrifuge Modeling
}

\author{
A. Jiménez Márquez \\ Instituto Andaluz de Investigación y Formación \\ Agraria, Pesquera, Alimentaria y de la Producción \\ Ecológica (IFAPA), Junta de Andalucía \\ Centro 'Venta del Llano' \\ PO. Box 50, E-23620 Mengibar, Jaén. Spain
}

\author{
G. Beltrán Maza \\ Instituto Andaluz de Investigación y Formación \\ Agraria, Pesquera, Alimentaria y de la \\ Producción Ecológica (IFAPA), Junta de Andalucía \\ Centro 'Venta del Llano' \\ PO. Box 50, E-23620 Mengibar, Jaén, Spain
}

\begin{abstract}
Machine learning through the Artificial Neural Networks Supervised Learning technique was applied for modelling the clarification process of Virgin Olive Oil by a disk stack centrifuge equipment. The neural network obtained was trained and validated by a real database generated from different experiments to analyze the performance of the centrifuge. Compositional variability of oil, the water/oil relations flow rates, and both temperatures to the centrifuge inlet were the technological variables that were checked about the oil loss in the waters process outlet as parameter indicator of the effectiveness on solid-liquid-liquid separation. The results obtained in validation indicate a good predictive capacity, with a correlation coefficient $\mathrm{r}>0.85$ and an error of $0.30 \mathrm{~kg} \mathrm{~h}^{-1}$, which allows this neural model to become an excellent tool to optimize the centrifuge. A 'Simulink' model was designed for performance verification of the Network by checking the predictions obtained from another data set that not intervened in its build. A t-Test was applied and the results indicate they are not a significant difference between predicted and real mean values for $\mathrm{P}=0.05$ and 60 degrees of freedom.
\end{abstract}

\section{General Terms}

Machine learning, Process Optimization, Intelligent Controls.

\section{Keywords}

Machine Learning; Neuronal Network; Optimization; Olive oil elaboration process; Disk Stack Centrifuge.

\section{INTRODUCTION}

In the field of 'Artificial Intelligence,' the 'Machine Learning' is highlighting as an emerging discipline in automatic learning systems due to its ability to build algorithms capable of predicting future behaviors from the analysis and identification of existing patterns in a database. Different learning models are known [1][2], being the Artificial Neural Networks (ANN) one them of the best known and the supervised learning the technique which has shown to have good effectiveness on industrial process optimization [3][4][5] and in the agro-industrial process control [6][7][8][9].

On extra virgin olive oil (EVOO) elaboration process and before of storage, water and solid impurities must be removed from the oily to avoid loss of oil and quality by fermentation and oxidation processes. This cleaning operation is carried out in the clarification stage, being the vertical centrifugation, by a disc stack centrifuge (DSC), the most used oil clarification method in the most of the current oil mills [10]. However the separation effectiveness is conditioned by the EVOO characteristics and those technological variables that affect it, such they are the water:oil ratio and their temperatures. Because the heterogeneity with which the EVOO input to de DSC, is necessary an optimization by adjust these variables to reduce the oil loss in residual aqueous effluents and producing the least effects on their physic-chemical and organoleptic characteristics [11]. At present, these adjust are carried out occasionally, manual and subjectively from appreciation which mill technician performs in the visual inspections of the effluents or from the analytical results of these, which results in a working environment of great uncertainty.

This uncertainty and the non-linear relationships between the different technological variables suggest that the application of Artificial Intelligence, through the Artificial Neuronal Networks (ANN), can be a good tool for the characterization, modelling and optimization of this process stage. The capacity of ANN for learning and solving complex relationship cases from an appropriate set of experimental data is known and, in recent times, the olive oil sector has started to incorporate this technology in different facets of the EVOO process $[12][13][14][15][16][17][18]$.

In this paper, preliminary results are showed on the viability of neural networks for non-linear modelling of the EVOO cleaning process in a DSC using the 'black-box' model, through the supervised learning technique, with data obtained from trials carried out in an olive mill, and with the aim of obtaining a model to be able to predict the separation efficiency of the machine through the loss of oil in the aqueous effluent.

\section{METHODOLOGY}

\subsection{Equipment}

The experiments were carried out in the experimental oil mill (Pieralisi, Zaragoza, Spain) of IFAPA Centro 'Venta del Llano' [11]. This is a continuous two-three phases EVOO elaboration process with a disk stack vertical centrifuge for oil cleaning. The DSC has a nominal capacity of $10 \mathrm{~m}^{3} / 24 \mathrm{~h}$, working at $8000 \mathrm{rpm}$ and equipped with a double-cone shape stack of 88 disks with a $202 \mathrm{~mm}$ diameter base, $59.24 \mathrm{~mm}$ of height, and $48.68^{\circ}$ disk slope. The water-oil interface was set with a $101 \mathrm{~mm}$ gravity disc. A temperature sensor, one flow meter, and one electrical valve controlled the water inlet to DSC. The oil inlet was monitored only by a temperature sensor, is not was automatized and the flow was irregularly depending on the outlet from the horizontal screw solid bowl 
(HSSB) or decanter. Sensors and actuators were integrated into an S.C.A.D.A. control software.

\subsection{Sampling and process data}

Samples of oil inlet and outlet and samples of process water outlet from DSC were taken by triplicate (samples process water inlet to DSC not were taken, they were drinking water). These samplings were carried out over several months and two olive crop years. The working condition for the relations of water-oil flow and temperatures were modified for each experiment. Different relationship of flow water:oil to inlet $(0: 1$ to $3: 1)$ and temperatures of both, were checked to analyze the effect on oil loss in process water outlet from DSC ( $\alpha \mathrm{w})$, expressed as $\mathrm{kg} \mathrm{h}-1$.

The samples and process data (flows and temperatures) were taken when the DSC operating conditions for each experiment were stabilized. The samples were analyzed immediately by the respective analytical procedures [13][14]. Moisture (Mo) and impurities solids (Io) were the parameters analyzed for the oils inlet, fat $(\mathrm{Fw})$ and solid matter $(\mathrm{Sw})$ for the outlet process water. From SCADA, flow (Qw) and temperature (Tw) of inlet water, flow (Qo) and temperature (To) of inlet oil, flow (Qwo) of outlet process water, flow (Qoo) of outlet oil, were the process data taken. The aw parameter was calculated from $\mathrm{Fw}(\%, \mathrm{w} / \mathrm{v})$ and $\mathrm{Qwo}(\mathrm{L}$ h-1).

A total of 74 experiments were carried out that they have provided a set of 225 samples. 128 samples (first and third repetitions) were applied to training and testing the ANN built, 64 samples (second repetition) for validation and rest 33 were used in the Simulink-simulator for checking performance. Table 1 shows the time sheet of the sampling process during DSC working. During each experiment one hour-long: 5 minute for internal cleaning by a solids discharge, 40 minute for DSC stabilization in new operating conditions, 2:30 minute for take sample 1,2:30 minute of wait, 2:30 minute for take sample 2 , other 2:30 minute of wait, and 2:30 minute for take sample 3 with other 2:30 minute of wait, previous before starting the work cycle again.

Table 1. Time Sheet on DSC sampling operations

\begin{tabular}{|c|c|c|c|c|c|c|c|c|c|c|c|c|}
\cline { 2 - 9 } \multicolumn{1}{c|}{} & \multicolumn{7}{c|}{1 hour } \\
\hline $\begin{array}{c}\text { Solids } \\
\text { discharge }\end{array}$ & & & & & & & & & & & & \\
\hline $\begin{array}{c}\text { Operating } \\
\text { conditions } \\
\text { stabilization }\end{array}$ & & & & & & & & & & & & \\
\hline Take sample 1 & & & & & & & & & & & & \\
\hline Take sample 2 & & & & & & & & & & & & \\
\hline Take sample 3 & & & & & & & & & & & & \\
\hline
\end{tabular}

\subsection{ANN build}

The ANN was built by using the Matlab software [19] and ANN tool, which provides the algorithms to carry out a supervised learning based on an algorithm of descending gradients type 'feed-forward-back-propagation'. The number of hidden layer (1-3), neurons per layer (15-25), transfer function between layers ('tansig','logsig','purelin'), training algorithms ('trainrp', 'trainlm', 'traingda') and number of iterations $(50-1000)$ were the parameters tested to find the best topology that allows predictions with a high level of lineal correlation coefficient (r), between the reference data and those predicted, with the least value of root mean of squared error (RMSE) on validation.

A simple virtual DSC simulator, designed by 'SimulinkMatlab' software [20], was developed for testing the performance of the ANN built. The 'gensim' function was used to translate the 'Net' obtained to a 'Simulink-block' who was connected with the appropriate blocks to generate this simulator. The predictions obtained from the 33 samples, who have not participated in build the ANN model, were tested by a t-Test (prediction vs real) to analyze the statistical significance of the differences found, rejecting the null hypothesis (prediction and real are different) when the value of $t_{s}$ is greater than the $t_{\alpha}$ of the Student table for $n-1$ degree of freedom and 95\% interval of confidence:

$\mathrm{t}_{\mathrm{s}}=\mathrm{D}_{\mathrm{m}} /(\mathrm{Std} / \sqrt{ } \mathrm{n})$

on Dm is the mean of differences between pairs of values, Std is the standard deviation of the differences, and $n$ the number of pairs of values.

\section{RESULTS AND DISCUSION}

\subsection{The used variables for network}

As it is well known, to design and build an ANN based on learning supervised is necessary to operate with real experimental data that allow them to learn and predict in reasonable agreement with the education received. For the case of this paper, the data obtained from the experiment designed has generated a data-base with a large amount of information about the behavior of the DSC in the different situations tested. One fact observed from these data, which agree with the visual observation, is that this process is submitted to a considerable degree of perturbations, ones due to the compositional characteristics of the oil inlet, others by the different technological variables that have to be handled in DSC accord with the separating efficiency criteria.

The flow and the compositional characteristics of oil inlet are non-uniform due to the characteristics of the olive fruits that they are processed through de HSSB. Water content and organic solid content are variables at each time, affecting the separation performance. During the process, the DSC separates the solids from the oil, a migration of water contained on oil to the process water added is produced and diffusion of the hydrosoluble compounds present on oil to water is carried out. These mass transfer processes have a direct relationship with the effectiveness of the machine and the intensity with which they manifest will depend on operating variables that can be regulated and optimized to achieve the most appropriate DSC performance.

Traditionally, to solve the problem a simultaneously water addition is carried out at flow and temperature higher to the oil. However, this high water:oil flow and temperature relations are the primary causes of EVOO loss quality, high production of effluents contaminant and high loss of oil in aqueous effluents [10][11][21]. In Figure 1 can be seen as, in general, the $\mathrm{I}_{\mathrm{o}}$ content and $\mathrm{Q}_{\mathrm{w}} / \mathrm{Q}_{\mathrm{o}}$ relation have a very important relationship with the DSC performance, through $\alpha_{w}$. This suggests that the first step to intelligent optimization of DSC is the adequacy of technological variables to characteristics of oil inlet. 


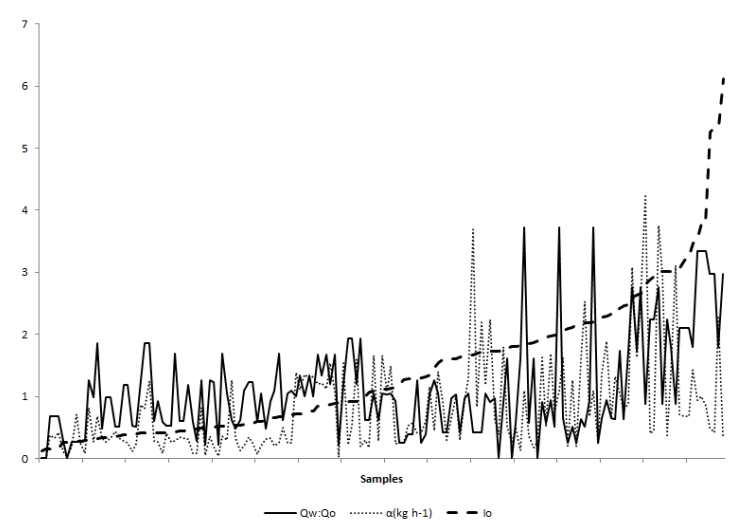

Fig 1: Graphical plot of values of: loss of oil in water outlet DSC $\left(\alpha_{w}\right)$, in $\mathrm{kg} \mathrm{h}^{-1}$; solid impurities in oil inlet DSC (Io), in \%; water:oil flow relation inlet $\operatorname{DSC}\left(Q_{w}: Q_{0}\right)$

From the control process point view, the centrifugal separation on DSC can be assimilated to a type of MISO control (multiple input and simple output). As shown in Figure 2 can be considered three types of variables: manipulable inputs, non-manipulable inputs and target output. The manipulable variables are made up of those variables that affect the response of the system and that can be optimized. The water and oil flow at CV inlet as well as their respective temperatures $\left(\mathrm{Qw}, \mathrm{Qo}, \mathrm{Tw}\right.$ and $\left.\mathrm{T}_{\mathrm{o}}\right)$ can be monitored and regulated, and their influence on the characteristics of the oil is known [1] [15]. There is another manipulable variable such as the gravity disk, which affects to the outlet the heavy phase by modification of diameter. The hole diameter handling is very difficult because it is necessary to stop the machine and must take disassembly to disk change, so it is considered appropriate to keep it fixed on modelling. Previously has been optimized and the value was established in the diameter that defines the optimal interior interface radius of the water:oil, and a compromise between purifying and concentrator actions was taken on.

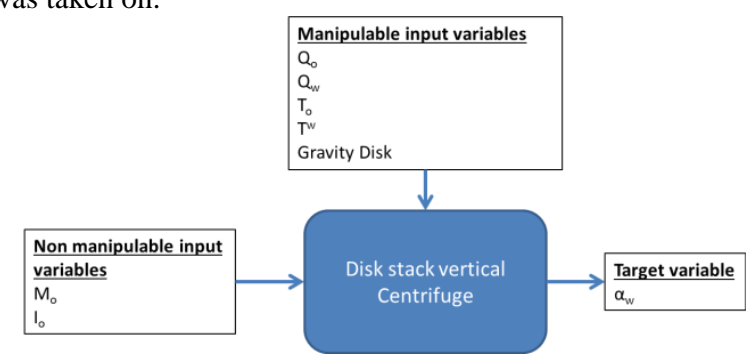

Fig 2: A graphical scheme of the main process variables that influence the operation of a disk stack centrifuge.

(Significate of abbreviates are indicated in the text)

As for the non-manipulable input variables, these are constituted by the compositional characteristics of oil inlet: Moisture $\left(\mathrm{M}_{\mathrm{o}}\right)$ and Solid impurities (Io) that cannot be controlled because their values, at the outlet from HSSB, are dependents on olive fruit and the treatments carried out in the previous stages of EVOO elaboration process.

The target output was defined, for this paper, by the most usually employed parameter on the mill: oil content in process water outlet $(\alpha \mathrm{w})$. That is the main indicator to checking the DSC performance by the loss of oil on residual water. For high $\alpha \mathrm{w}$ values significant oil leaks can be expected.

In Table 2 are summarized the values maximum, minimum, mean and ranges of these variables, obtained from the experimental tests carried out, those were split into training and validation set. A high variability can be seen which can ensure an ANN model reliable.

Table 2. Statistical data of the different variables used on ANN build and splitted on the training and validation set.

\begin{tabular}{|c|c|c|c|c|c|c|c|}
\hline \multirow{2}{*}{} & \multicolumn{7}{|c|}{ Training set (128) } \\
\cline { 2 - 8 } & $\begin{array}{c}\mathrm{M}_{\circ} \\
(\%)\end{array}$ & $\begin{array}{c}\mathrm{I}_{\circ} \\
(\%)\end{array}$ & $\begin{array}{c}\mathrm{Q}_{0}(\mathrm{~kg} \\
\left.\mathrm{h}^{-1}\right)\end{array}$ & $\begin{array}{c}\mathrm{Q}_{\mathrm{w}}(\mathrm{kg} \\
\left.\mathrm{h}^{-1}\right)\end{array}$ & $\begin{array}{c}\mathrm{T}_{\circ} \\
\left({ }^{\circ} \mathrm{C}\right)\end{array}$ & $\begin{array}{c}\mathrm{T}_{\mathrm{w}} \\
\left({ }^{\circ} \mathrm{C}\right)\end{array}$ & $\begin{array}{c}\alpha_{\mathrm{w}}(\mathrm{kg} \\
\left.\mathrm{h}^{-1}\right)\end{array}$ \\
\hline Max & 23.7 & 6.12 & 432 & 564 & 35.5 & 39.5 & 4.23 \\
\hline Min & 0.86 & 0.12 & 84 & 0 & 22.5 & 13.4 & 0.01 \\
\hline STD & 4.66 & 1.16 & 87 & 127 & 3.9 & 6.8 & 0.82 \\
\hline & 6.76 & 1.40 & 252 & 238 & 28.7 & 22.5 & 0.80 \\
\cline { 2 - 9 } & $\begin{array}{c}\mathrm{M}_{\circ} \\
(\%)\end{array}$ & $\begin{array}{c}\mathrm{I}_{\circ} \\
(\%)\end{array}$ & $\begin{array}{c}\mathrm{Q}_{\circ}(\mathrm{kg} \\
\left.\mathrm{h}^{-1}\right)\end{array}$ & $\begin{array}{c}\mathrm{Q}_{\mathrm{w}}(\mathrm{kg} \\
\left.\mathrm{h}^{-1}\right)\end{array}$ & $\begin{array}{c}\mathrm{T}_{\circ} \\
\left({ }^{\circ} \mathrm{C}\right)\end{array}$ & $\begin{array}{c}\mathrm{T}_{\mathrm{w}} \\
\left({ }^{\circ} \mathrm{C}\right)\end{array}$ & $\begin{array}{c}\alpha_{\mathrm{w}}(\mathrm{kg} \\
\left.\mathrm{h}^{-1}\right)\end{array}$ \\
\hline Max & 21.1 & 5.32 & 432 & 564 & 35.5 & 39.5 & 4.22 \\
\hline Min & 0.97 & 0.15 & 84 & 0 & 22.5 & 13.4 & 0.01 \\
\hline Mean & 6.63 & 1.34 & 261 & 228 & 28.6 & 23.0 & 0.82 \\
\hline STD & 4.49 & 1.02 & 95 & 125 & 3.9 & 7.0 & 0.82 \\
\hline
\end{tabular}

\subsection{The network}

For modelling DSC the 'black-box' model was used. The neuronal net was obtained from the relationships established between the variables measured at the input and the variables measured at the output from the experimental data. Thus, the input vector to the neural network ' $p$ ' was made up of the variables: Mo, Io, Qo, Qw, To and Tw, while the vector output from the network ' $t$ ' was the variable: $\alpha w$.

The building of ANN was based on the use of training algorithms based on the back-propagation of errors ('Newff' function), with the input data normalized in the range ' $-1+1$ ' using the 'premnmx' function of the Matlab software [18]:

$$
\mathrm{pn}=[2 *(\mathrm{p}-\mathrm{pmin}) /(\mathrm{pmax}-\mathrm{pmin})-1)
$$

on $\mathrm{p}$ is the input vector, $\mathrm{pn}$ the normalized values of $\mathrm{p}$, and pmin to pmax the minimal and maximal values on 'p'vector.

The search for the best model was carried out using a 'script' with which the vectors ' $p$ ' and ' $t$ ' are loaded, normalized, splits into their training and validation sets, train the network, its validation and obtain the linear correlation between the actual values and predicted values on validation.

The training stage applies the Matlab algorithm:

newff(PR,[S1 S2.SN1],\{TF1 TF2...TFN1\},BTF,BLF,PF (3)

where: PR -- R x 2 matrix of min and max values for $\mathrm{R}$ input elements; $\mathrm{Si}$-- Size of ith layer, for N1 layers; TFi -- Transfer function of ith layer; BTF -- Backpropagation network training function; BLF -- Backpropagation weight/bias learning function; PF -- Performance function.

For the learning function was used, by defect, the gradient descent with momentum weight and bias learning ('learngdm'). The ANN performance was measured by the Root Mean Squared normalized Error performance function (RMSE). The rest of the algorithm elements (number of layers, neurons per layer and iterations) were modified in each script execution until finding the combination that was 
provided the best indicators of the model: high lineal coefficient of correlation and low RMSE.

The function used on script phase validation was:

$$
\mathrm{t}=\operatorname{sim}(\text { net }, \mathrm{x})
$$

where: net, is the trained neural network; $\mathrm{x}$, is the input vector with the data for validation; $t$, are the values of the output vector predicted by the network.

Finally, the function:

$$
[\mathrm{m}, \mathrm{b}, \mathrm{r}]=\operatorname{pos} \operatorname{treg}(\mathrm{t}, \mathrm{tval})
$$

was used to analyze the linear correlation ( $r$ ) between the real values (tval) and predicted values on validation ( $t$ ) those restored to original format by function 'postmnmx':

$$
\mathrm{t}=\left[0.5^{*}(\mathrm{tn}+1) *(\operatorname{tmax}-\mathrm{tmin})\right]+\mathrm{tmin}
$$

on $\mathrm{t}$ is the target values restored, th the normalized values predicted by the network, and tmin to tmax the minimal and maximal values of vector target $t$.

How the process is trial-error, the 'script' was started using a topology already used in other papers [17], which was modified according to the results that were obtained. Table 3 shows a resume of best correlations in training for an initial topology of 18/25/1 with 'Tan-Sigmoid function' (Tsg) as inter-layer transfer functions.

Table 3. The best lineal correlation in training from different 'training functions' and initial topology of 18/25/1 neurons by layer.

\begin{tabular}{|c|c|c|}
\hline Iterations & $\begin{array}{c}\text { Training } \\
\text { Function }\end{array}$ & $\begin{array}{c}\text { Training } \\
\text { correlation } \\
(\mathrm{r})\end{array}$ \\
\hline 1000 & Tgdx & 0.854 \\
\hline 1500 & Tgda & 0.813 \\
\hline 800 & Toss & 0.860 \\
\hline 1000 & Trp & 0.877 \\
\hline 390 & TIm & 0.848 \\
\hline
\end{tabular}

Based on these initial results, the Trp training function was chosen, next iterations and neurons by layer were checked to improve the correlation index.

The result was obtaining the best ANN model which topology is shown in Table 4. As can be seen, the best model requires three layers, the input layer with 15 neurons, a hidden layer with 20 neurons and the output layer with 1 neuron. The best inter-layer transfer functions are the 'Tan-Sigmoid function' (Tsg) or 'Hiperbolic tangent sigmoid', which presents a temporal progression, between normalized values $-1+1$, with a sigmoidal characteristic curve, similar to the behavior curves that appear in many natural processes and learning processes, and whose function can be expressed by the equation:

$\operatorname{tansig}(n)=2 /(1+\exp (-2 * n))-1$

Table 4. Topologie final of the ANN build

\begin{tabular}{|c|c|c|c|}
\hline \multicolumn{4}{|c|}{ Training } \\
\hline NT/NV & Layer and & Layer & Training \\
\hline
\end{tabular}

\begin{tabular}{|c|c|c|c|}
\hline & neurons & $\begin{array}{c}\text { Transfer } \\
\text { functions }\end{array}$ & function \\
\hline $128 / 64$ & $15 / 20 / 1$ & Tsg/Tsg/Tsg & Trp \\
\hline \multicolumn{4}{|c|}{ Validation } \\
\hline \multirow{4}{*}{} & Iterations & $r$ & RMSE $\left(\mathrm{kg} \mathrm{h}^{-1}\right)$ \\
\cline { 2 - 4 } & 500 & 0,888 & 0.31 \\
\cline { 2 - 4 }
\end{tabular}

As activation function the most suitable solution was the 'Resilient backpropagation' (trainrp), which is a network training function that performs convergence more quickly, reducing training needs. The number of iterations used to achieve a MSE error $<0.01$ was 500 , more iterations become in an overfitting of the model (Table 5).

Table 5. Lineal correlation in validation by iterations for 15/20/1 neurons by layer, 'Tsg' as transfer functions and 'Trp' as training function.

\begin{tabular}{|c|c|}
\hline $\begin{array}{c}\text { № } \\
\text { iterations }\end{array}$ & $\begin{array}{c}\text { Lineal } \\
\text { correlation } \\
(r)\end{array}$ \\
\hline 350 & 0.848 \\
\hline 500 & 0.865 \\
\hline 1000 & 0.888 \\
\hline 1500 & 0.871 \\
\hline 2000 & 0.843 \\
\hline
\end{tabular}

The ANN model validation, through the $2^{\circ}$ repetition from each experiment, has allowed to obtain a high significant lineal correlation with value of $\mathrm{r}=0.888$ and prediction error of $\mathrm{RMSE}=0.31 \%$. In Figure 3 is shown a graphical comparison between real data and those obtained by de ANN model, on this validation stage, which suggests an adequate behavior of the model for $\alpha_{w}$ values predictions.

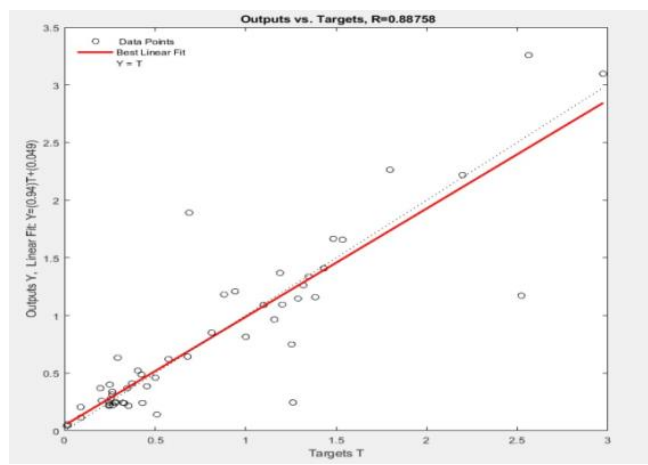

Fig 3: Graphical plotting of linear correlation between values from ANN prediction (T) and real values (y) on the validation stage of network build, from 'nntraintools' of Matlab

This ANN model was translated to 'Simulink' environment by the 'gensim' Matlab function. A simple DSC simulator was designed (Figure 4) to display the $\alpha_{\mathrm{w}}$ prediction values from different input values of the technological variables supplied by the rest of 33 experiments not employed on training and validation of the ANN model. The input variables are defined in 'constant' blocks, normalized at -1 to +1 by 'premnmx' 
costumer blocks and input to Neural Network by a multiplexer. The predicted value is restored by 'postmnmx' costumer block and result monitored by a display block and plotted by a 'scope' block. A block sends different $\mathrm{Q}_{\mathrm{w}}$ in a sequence of each100 unit times

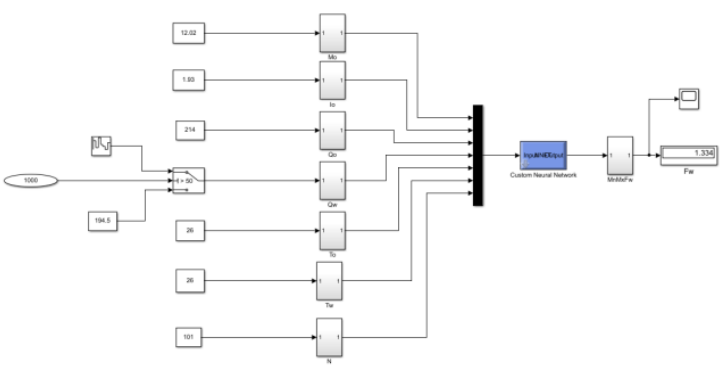

Fig. 4: Graphical scheme of simulator created by 'Simulink'.

A t-Test for comparison of means with unequal variances, between $\alpha_{\mathrm{w}}$ simulated and real values, indicate that the calculated value of $t_{\text {cal }}=0.691$ is less than the tabulated values of $\mathrm{t}_{\text {theor }}=1.671$, for $\mathrm{P}=0.05$ and 60 degrees of freedom. The mean values obtained using the ANN model, do not differ significantly from the real values obtained by laboratory analytical (Table 6)

Table 6. Results of t-Test for two samples and unequal variances

\begin{tabular}{|c|c|c|}
\cline { 2 - 3 } \multicolumn{1}{c|}{} & $\begin{array}{c}\alpha_{\mathrm{w}}(\text { Real } \\
\text { value })\end{array}$ & $\begin{array}{c}\alpha_{\mathrm{w}} \text { (ANN } \\
\text { predicted) }\end{array}$ \\
\hline Mean & 0.274848485 & 0.236969697 \\
Variance & 0.062250758 & 0.036990218 \\
Observations & 33 & 33 \\
Degrees of freedom. & 60 & \\
$\begin{array}{c}\text { Statistical t } \\
\text { Critical value of } \mathrm{t} \\
(\mathrm{P}=0.05))\end{array}$ & 0.690728747 & \\
\hline
\end{tabular}

In Figure 5 an example register of simulator prediction comportment for different Qw values inlet as setpoint is showed.

In this example, for the rest variables constant: $\mathrm{Qo}=215 \mathrm{~L} \mathrm{~h}-1$, $\mathrm{Mo}=12.02 \%, \mathrm{Io}=1.93 \%$, To and $\mathrm{Tw}=26^{\circ} \mathrm{C}$, changes in water inlet flow values predict the $\alpha_{\mathrm{w}}$ values shows in Table 7 , accord with the graphical displayed. In general, can be seen that higher level of $\mathrm{Q}_{\mathrm{w}}$ lead to greater oil losses that those levels that make the $\mathrm{Q}_{\mathrm{w}} / \mathrm{Q}_{\mathrm{o}}$ relation less than one.

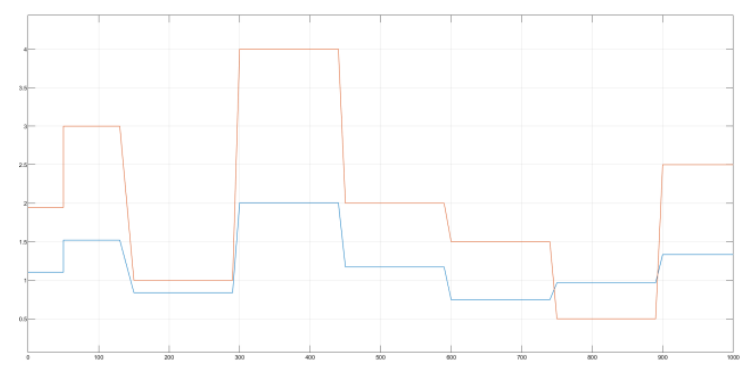

Fig 5: Graphical plotting on predicted values of $\boldsymbol{\alpha}_{\mathrm{w}}$ (blue) obtained from simulator when a sequential of different $Q_{w}$ values $(\mathrm{red} / 100)$ were applied
Thus, through this simulator, the ANN built allows to define, in real time in the oil mill, the most appropriate $\mathrm{Q}_{\mathrm{w}} / \mathrm{Q}_{\mathrm{o}}$ ratio to minimize oil losses on clarification stage.

\section{CONCLUSIONS}

By using the ANN technique is feasible to develop virtual models that allow simulating the performance of a vertical centrifuge in the clarification stage of the EVOO elaboration process. These models can be developed to predict, with sufficient guarantee, the effectiveness of the separative function through the loss of oil in the aqueous effluent outlet. Therefore, DSC modeling using ANN is shown as an important tool for optimizing this stage of the EVOO process since, by obtaining immediate and real-time predictive results, can allow on-line adjustment of machine operating conditions depending on the characteristics of the oil inlet. Furthermore, it becomes essential the development and use of ANN models for the design of intelligent controllers for optimization of this stage. An ANN model, as a virtual plant, facilitates the experimental study of these at the laboratory level because can be tuned them before their scaling to an industrial level since a real study would imply the realization of a work program that would originate a large number of samples, consuming a large amount of time, human resources and raw material such that the duration of the tests could originate a lack of homogeneity on oil batches, as well as significant economic losses.

Table 7. Values of $\alpha_{w}$ from ANN predicted vs real values obtained from graphic of Figure 5.

\begin{tabular}{|c|c|c|c|}
\hline Times & $\begin{array}{c}\text { Qw } \\
\text { setpoint } \\
\left(\mathrm{L} \mathrm{h}^{-1}\right)\end{array}$ & $\begin{array}{c}\alpha_{\mathrm{w}}(\mathrm{ANN} \\
\text { predicted }) \\
\left(\mathrm{Kg} \mathrm{h}^{-1}\right)\end{array}$ & $\begin{array}{c}\alpha_{\mathrm{w}} \text { (Real } \\
\text { value }) \\
\left(\mathrm{Kg} \mathrm{h}^{-1}\right)\end{array}$ \\
\hline $0-50$ & 195 & 1.10 & 1.51 \\
\hline $50-150$ & 300 & 1.52 & 1.78 \\
\hline $150-300$ & 100 & 0.84 & 0.78 \\
\hline $300-450$ & 400 & 2.10 & 1.98 \\
\hline $450-600$ & 200 & 1.17 & 1.12 \\
\hline $600-750$ & 180 & 0.75 & 1.05 \\
\hline $750-900$ & 50 & 0.97 & 0.82 \\
\hline $900-1000$ & 250 & 1.13 & 1.47 \\
\hline
\end{tabular}

Finally, the performance of ANN models is only ensured when a data-base with a large amount of information about the behavior of the DSC if available, because their responses will depend on how they are educated. So, is essential that this database must be reliable, free spurious data, and cover all aspects of machine behavior by data filtering, experimental design and appropiate choice of process variables, in order to obtain consistent algorithms over time, that can respond to unexpected future situations.

\section{ACKNOWLEDGMENTS}

This work was supported by P10-AGR6429 Excellent Project: 'Modelling and Optimization of Virgin Olive Oil Elaboration Process. Project I', financed by 'Ministerio de Ciencia e Innovación' and 'Consejería de Economía, Innovación y Ciencia de la Junta de Andalucía' (Spain) and Proyect IFAPA.PPVA:AVA2016.12 'Virgin Olive Oil Quality and Safety. Objetiv 2: Intelligent Control in Virgin Olive Oil 
elaboration process', $80 \%$ financed by FEDER (FEDER Andalucía 2014-2020). Special thanks to Juan Torres and Justo Cardenas by their collaborations in oil mills and all the laboratory personnel.

\section{REFERENCES}

[1] P. Harrington. Machine Learning in action. Manning Publication Co., Shelter Island. New York. 2012.

[2] K.G. Liakos, P. Busato, D. Moshou, S. Pearson, D. Bochtis, Machine Learning in Agriculture: A Review, Sensor, 18 (2018) 2674. 2018.

[3] K.J. Hunt, D. Sbarbaro, R. Zbikowski, P.J. Gawthrop, Neural network for control system, Automatica, 28 (1992)

[4] S. Linko, Y.H. Zhu, P. Linko, Applying neural networks as software sensors for enzyme engineering, Trends in Biotechnology, 17(4) (1999) 155-162. DOI:10.1016/S0167-7799(98)01299-2.

[5] C. Ahilan, J.E. Raja Dash, K. Somasundaram, N. Sivakumara. Performance assessment of heat exchanger using intellingent decision making tools. Applied Soft $\begin{array}{llll}\text { Computing } & 26 & \text { (2015) }\end{array}$ DOI:10.1016/j.asoc.2014.10.018.

[6] J.M. Zamarreño, P. Vega, Identification and predictive control of a melter unit used in the sugar industry, Artificial Intelligence in Engineering, 11 (4) (1997) 365373.

[7] I. Ceylan, I. Aktaş, Modeling of a hazenut dryer assisted heat pump by using artificial neural networks, Applied Energy, 85 (2008) 841-854.

[8] V.C. Mircea, R. Raluca, P.A. Şerban, Neural networks based model predictive control of the drying process In: Andrzej Kraslawski and Ilkka Turunen (Eds), Computer Aided Chemical Engineering, Elsevier,2003, pp. 389394.

[9] J.P. Steyer, I. Queinnec, D. Simoes, Biotech: a real-time application of artificial intelligence for fermentation processes, Control Eng. Practice 1(2) (1993) 315-321.

[10] M. Uceda, G. Beltrán, A. Jiménez, Olive oil extraction and quality, Grasas Aceites 57 (2006) 25-31.

[11] A. Jiménez, M. Hermoso, M. Uceda, Extraction of virgin olive oil by two-phases continuous system. Influence of different variables of the process on certain parameters related to oil quality, Grasas y Aceites 4-5 (1995) 299303.

[12] [R. Furferi, M. Carfagni, M. Daou, Artificial neural network software for real-time estimation of olive oil qualitative parameters during continuous extraction, Computers and Electronics in Agriculture 55(2) (2007) 115-131. DOI:10.1016/j.compag.2006.12.006.

[13] A. Jimenez, G. Beltran, M.P. Aguilera, M. Uceda, A sensor-software based on artificial neural network for the optimization of olive oil elaboration process, Sensors

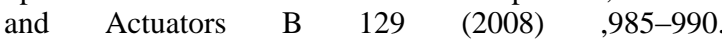
DOI:10.1016/j.snb.2007.09.030.

[14] A. Jiménez, M.P. Aguilera, M. Uceda, G. Beltrán, Neural network as tool for virgin olive oil elaboration process optimization, Journal of Food Engineering 95 (2009) 135-141. DOI:10.1016/j.jfoodeng.2009.04.021.

[15] E. Funes, Y. Allouche, G. Beltrán, A. Jiménez, A Review: Artificial Neural Netwokrs as Tool for Control Food Industry Process, Journal of Sensor Technology 5 (2015) 28-43.. DOI:10,4236/jst2015,51004.

[16] Y. Allouche, E. Funes, G. Beltrán, A. Jiménez, Near infrared spectroscopy and artificial neural network to characterise olive fruit and oil on line for process optimization, Journal of Near Infrared Spectroscopy 23 (2015) 111-121. DOI: 10,1255/jnirs.1155.

[17] E. Funes, Y. Allouche, G. Beltrán, M.P. Aguilera, A. Jiménez, Predictive ANN models for the optimization of extra virgin olive oil clarification by means of vertical centrifugation. Journal of Food Process Engineering 41(1) (2017) 1-8. DOI:10,1111/jfpe,12593.

[18] E. Funes, Y. Allouche, G. Beltrán, M.P. Aguilera, A Jiménez, A predictive artificial neural network model as a simulator of the extra virgin olive oil elaboration process. Journal of Near Infrared Spectroscopy 25(4) (2017) 278-285. DOI: 10,1177/0967033517725639.

[19] H. Demuth, M. Beale, Neural Network Toolbox. For use with MATLAB. User`s Guide Rev 4. (2001).

[20] Matlab 7.0, The MathWorks Inc., Natick, MA, USA.

[21] P. Masella, A. Parenti, P. Spugnoli, L.Calamai, Vertical centrifugation of virgin olive oil under inert gas, Eur J Lipid Sci Technol 114 (2012) 1094-1096 